\title{
DERMATOLOGIA NA ATENÇÃO PRIMÁRIA À SAÚDE: UMA REVISÃO DE LITERATURA
}

\author{
DERMATOLOGY IN PRIMARY HEALTH CARE: A LITERATURE \\ REVIEW
}

\author{
Bruna Caldas Campos ${ }^{1}$ \\ Luciana Modesto de Brito $^{2}$ \\ Cícera Amanda Mota Seabra ${ }^{3}$ \\ Ankilma do Nascimento Andrade Feitosa ${ }^{4}$
}

\begin{abstract}
RESUMO: Introdução: A formação do médico generalista requer a aquisição de diversos conhecimentos na área da saúde. Uma das especialidades, de extrema importância, é a Dermatologia, a qual requer um treinamento e estudo direcionados. A graduação médica, por vezes, é deficiente em aprofundar o estudo em áreas específicas. Isso pode dificultar a prática do médico na Atenção Primária, que deveria focar a sua atenção na promoção e prevenção de doenças, e quando isso
\end{abstract}

\footnotetext{
1 Médica. Residente em Saúde da Família e Comunidade da Faculdade Santa Maria, Cajazeiras, Paraíba.

2 Mestra em Sistemas Agroindustriais pela UFCG. Pós-graduanda em Cardiologia. Graduada em Medicina pela Faculdade de Medicina Nova Esperança FAMENE (2011). Especialização em Preceptoria de Residência Médica no SUS pelo Hospital Sírio Libanês. Especialização em Medicina Intensiva pela Faculdade Redentor, Rio de Janeiro. Atualmente Médica Intervencionista do Serviço de Atendimento Móvel de Urgência (SAMU), Médica diarista do Hospital Padre Alfredo Barbosa), atua na Faculdade Santa Maria como Docente do curso de medicina, coordenadora do Internato Médico e responsável técnica da Clínica Santa Maria, Cajazeiras, Paraíba.

3 Médica com graduação em Medicina pela Universidade Federal de Campina Grande (2008). Trabalhou por 8 meses como médica contratada do Programa de Saúde da Família da Prefeitura Municipal de Itapipoca de maio a dezembro de 2008. Concluiu Residência Médica pelo Programa de Pós-Graduação em Residência de Medicina de Família e Comunidade do Sistema Municipal de Saúde Escola da Prefeitura Municipal de Fortaleza com duração de 2 anos, tendo iniciado em janeiro de 2009 e concluído em janeiro de 2011. Possui Título de Especialista em Medicina de Família e Comunidade pela Associação Médica Brasileira. Exerceu a função de Médica de Família e Comunidade concursada do Programa de Saúde da Família da Prefeitura Municipal de Aurora-CE desde janeiro de 2011 a março de 2019, atualmente é médica concursada da Prefeitura Municipal de Barbalha-CE e, também, é supervisora do Programa Mais Médicos para o Brasil desde janeiro de 2014. Desde fevereiro de 2016 exerce a função de professora contratada da Faculdade Santa Maria (FSM) em Cajazeiras - PB das disciplinas Programa de Aprendizagem da Atenção Básica (PAAB) IV e VIII, Semiologia e Saúde do Idoso, e está na coordenação do Programa de Residência de Medicina de Família e Comunidade em Cajazeiras-PB.

${ }^{4}$ Pós-doutorado pela Universidade Federal de Campina Grande. Doutora em Ciências da Saúde pela Faculdade de Medicina do ABC. Docente da Faculdade Santa Maria, Cajazeiras, Paraíba.
} 
não ocorre, um diagnóstico é postergado, trazendo danos para o paciente e para o sistema público que, a depender da evolução da doença, arcará com gastos maiores como exames, encaminhamentos, medicações e internamento hospitalar. Objetivo: Identificar a importância de conhecer as principais doenças de pele na Atenção Primária. Método: Estudo bibliográfico com abordagem qualitativa realizada durante o período de setembro de 2021 a janeiro de 2022 pelas bases de dados Literatura e National Library of Medicine (PubMed), Biblioteca Nacional em Saúde (BVS) e Scientific Electronic Library Online (SciELO). Como critérios de inclusão foram utilizados artigos nacionais e internacionais que estivessem disponíveis nas bases de dados citadas, com resumos disponíveis e artigo de disponibilização gratuita, publicados entre o ano de 2016 a 2021. Resultados: Inúmeros benefícios são alcançados quando ocorre o diagnóstico precoce das doenças de pele. Quando o médico está apto para abordagem diagnóstica de uma afecção de pele, na Unidade Básica de Saúde, o número de encaminhamentos para especialistas diminui, assim como a solicitação de exames e gastos com tratamento quando a doença é descoberta no início. Conclusão: Portanto, conclui-se que a atenção primária à saúde é o ponto de acesso preferencial do sistema de saúde e, se o médico apresenta conhecimento, resolutividade e qualificação, é capaz de resolver a maioria das queixas dermatológicas comumente apresentadas.

Palavras-chave: Dermatologia. Doenças de Pele. Afecções cutâneas. Atenção Primária à Saúde.

ABSTRACT: Introduction: The training of the general practitioner requires the acquisition of diverse knowledge in the health area. One of the extremely important specialties is Dermatology, which requires training and targeted study. The medical offer is sometimes in-depth to deepen the study in specific areas. It can make it difficult to practice in Primary Care, which must focus its attention on the promotion and not prevention of diseases and when this occurs a diagnosis is postponed, with damages to the patient and to the public system that depends on the evolution of the disease, bears greater costs. such as exams, exams and internal medicines, medicines. Objective: To identify the importance of knowing the main diseases in Primary Care. Method: Bibliographic study with a qualitative approach carried out during the period from 2021 to January 2020 by the Literature and National Library of Medicine (PUB-MED), National Health Library (BVS) and Scientific Electronic Library Online (SciELO) databases. As well as free inclusion, national and international articles were used that immediately make available databases cited, with abstract and availability 16 between 2016 and 2021. Results: When the disease is available for medical approach, the diagnosis is easier for a skin detection in the Basic Health Unit, the number of treatments for specialists, as well as the need for exams and treatment expenses at a basic health unit, when late. Conclusion: it is concluded that health care is the point of preferential health care, if the doctor is resolute and qualified, is able to solve most dermatological complaints, preferential.

Keywords: Dermatology. Skin diseases. Skin disorders. Primary Health Care. 


\section{INTRODUÇÃO}

A pele é o maior órgão do corpo humano e um dos mais importantes sistemas de interação entre o meio interno e o meio externo (AZULAY, 2008). Desempenha algumas funções como proteção física, química e imunológica, manutenção da homeostasia e informação sensorial. As doenças da pele são muito frequentes nas consultas médicas, e têm se tornado um mal bastante comum que aflige a vida de milhões de pessoas atualmente. As doenças dermatológicas estão entre as queixas mais comuns em diversos serviços de saúde, incluindo aqueles da Atenção Primária à Saúde - APS (PIMENTEL, 2011; MINISTÉRIO DA SAÚDE, 2002; BERNARDES, 2015). Por ser queixa prevalente, e pela chance de tratamento ambulatorial ser bem sucedido, é necessário que os médicos da Atenção Primária estejam habilitados para o correto diagnóstico e tratamento do paciente, diminuindo assim o impacto na vida dos mesmos.

Alguns fatores estão envolvidos com a dificuldade na abordagem destas afecções, e entre eles merece destaque o isolamento da disciplina na graduação em Medicina, com dificuldade de integração curricular e carga horária diminuta. Em muitos cursos, observa-se um déficit no ensino da dermatologia, tanto teórico quanto prático, e médicos não especialistas acabam por se sentir despreparados para o enfrentamento das afecções da pele nas suas atuações práticas (BARBARULO, 2002). Essas limitações parecem se repetir também no âmbito da atenção primária, considerada como porta de entrada do sistema de saúde, trazendo dificuldades no diagnóstico e seguimento das afecções da pele (JANAUDIS, 2010).

A Estratégia Saúde da Família (ESF) já é uma realidade, com cerca de 31 mil equipes implantadas até abril de 2011 (MINISTÉRIO DA SAÚDE, 2011), e muitos esforços têm sido realizados para aprimorar este modelo. A Atenção Primária nesta política, supostamente, dará conta de cerca de $80 \%$ dos problemas de saúde da sua clientela adscrita, e para isto necessita do apoio de uma rede de saúde funcionante (MENDES, 2010). 
O uso de materiais educativos como recursos na educação em saúde vem crescendo, assumindo um papel de destaque no processo de ensino-aprendizagem (MOREIRA, 2003). Isso contribui, de forma significativa, para a formação médica, que atuará com uma carga de conhecimento necessária para abordagem diante de afecções dermatológicas.

Visto isso, o objetivo do presente artigo foi identificar a importância de conhecer as principais doenças de pele na atenção primária à saúde, além de desenvolver estratégias educativas para ajudar no diagnóstico de doenças cutâneas, e apresentar estratégias de adesão que podem ser adotadas pelas Unidades de Saúde da Família para identificação precoce de afecções na pele.

\section{MÉTODOS}

O estudo trata-se de uma revisão de literatura de caráter descritivo e explanatório, que envolveu como tema central a importância da dermatologia na atenção primária à saúde, com abordagem qualitativa realizada no período compreendido entre setembro de 2021 a janeiro de 2022.

A pesquisa exploratória visa proporcionar maior visão sobre o tema selecionado. Dedicando-se, dessa forma, ao levantamento bibliográfico e/ou documental (SANTOS; CANDELORO, 2006), que, por sua vez, são realizados a partir da pesquisa de referenciais teóricos já estudados e publicados em meios eletrônicos e escritos (GERHARDT; SILVEIRA, 2009).

Para Gil (2010), o objetivo do estudo da pesquisa descritiva é identificar as características de um determinado fenômeno, buscando obter opiniões, atitudes e crenças de uma população.

A revisão da literatura é uma análise bibliográfica mais detalhada a partir de estudos já publicados de um determinado assunto, envolve localizar, explorar, resumir e interpretar a investigação prévia com uma área do assunto (BENTO, 2012). 
Para busca de artigos foram utilizadas as bases de dados Literatura e National Library of Medicine (PubMed), Biblioteca Nacional em Saúde (BVS) e Scientific Electronic Library Online (SciELO).

Os Descritores em Ciências da Saúde (DeCS) utilizados foram Dermatologia, Atenção Primária à Saúde, Doenças de Pele, Doenças Cutâneas. Os critérios de inclusão contemplados foram artigos nacionais e internacionais que estivessem disponíveis nas bases de dados citadas, com resumos disponíveis e artigo de disponibilização gratuita, publicados entre o ano de 2016 a 2021. Os critérios de exclusão definidos foram artigos incompletos, teses e dissertações que não possuíam relação direta com a temática abordada.

Após estabelecidos os critérios, iniciou-se a coleta de dados, que consiste na aplicação dos descritores e critérios de inclusão e exclusão nas bases de dados, para selecionar e resumir um conjunto de dados que podem ser facilmente utilizados, após seleção, para compor a pesquisa.

Foram escolhidos os descritores e seu operador booleano: "Dermatologia and Atenção Primária", "Doenças de pele and Atenção Primária à Saúde".

\section{RESULTADOS E DISCUSSÃO}

Após cruzamento dos critérios de inclusão e descritores foram encontrados 01 artigo na BVS, 02 na SciELO e 50 na PubMed. Foram selecionados 18 artigos após leitura do título e resumo, superficialmente, para estudo aprofundado do conteúdo. Após esta etapa, 07 artigos foram inclusos na pesquisa por satisfazer os critérios de inclusão estabelecidos.

No Brasil, até o momento, são poucas as ferramentas elaboradas para subsidiar a abordagem das afecções da pele por profissionais não especialistas. Capacitações e treinamentos direcionados aos médicos da ESF não costumam contemplar aspectos da atenção a essas afecções, principalmente sob a ótica da prevenção destes agravos (CAMPOS, 2007). Uma abordagem integral na graduação de Medicina se mostra extremamente importante no auxílio do médico generalista. A 
inserção das especialidades nesta nova e necessária abordagem integral não é tarefa fácil, e implica na revisão dos métodos atuais de ensino e aprendizado desenvolvidos ao longo da graduação, e nos próprios serviços de atenção primária, considerados como portas de entrada para o sistema de saúde (JANAUDIS, 2010).

Foi possível observar que a adequada formação médica, permite o aumento da resolubilidade dos casos na Atenção Primária, diminuindo gastos, como encaminhamentos desnecessários, solicitação de exames e menor tempo de espera para tratamento de uma doença cutânea.

\section{CONCLUSÃO}

Diante do exposto, foi possível observar que é de extrema importância o conhecimento médico na área da Dermatologia, para que possa conduzir o paciente a um desfecho favorável de tratamento. Isso será possível se, além da inclusão de aprofundamento na disciplina durante a graduação, assim como cursos educativos para complementar a melhor formação do médico, e com isso seja possível o diagnóstico, tratamento e acompanhamento adequado do usuário que procura a Atenção Básica para resolução de suas queixas. Já que as mesmas impactam de forma significativa na vida das pessoas, causando, muitas vezes, sofrimento físico e psicossocial, além de trazer impactos significativos para o sistema público de saúde. 


\section{REFERÊNCIAS BIBLIOGRÁFICAS}

BARBARULO, A. et al. Evaluación de la capacidad diagnóstica de los médicos generales en dermatología. [s.l: s.n.]. Disponível em: <http://www.archivosdermato.org.ar/wpcontent/uploads/2020/02/52-3-111-115-2002.pdf>. Acesso em: 5 fev. 2022.

BERNARDES, C. A. et al. Diagnóstico e Condutas Dermatológicas em uma Unidade Básica de Saúde. Revista Brasileira de Educação Médica, v. 39, n. 1, p. 88-94, mar. 2015.

BRASIL. Departamento de Atenção Básica, Saúde da Família. Atenção Básica e a Saúde da Família. [s.I.] MINISTÉRIO DA SAÚDE, 2011.

CAMPOS, G. W. DE S.; DOMITTI, A. C. Apoio matricial e equipe de referência: uma metodologia para gestão do trabalho interdisciplinar em saúde. Cadernos de Saúde Pública, v. 23, n. 2, p. 399-407, fev. 2007.

JANAUDIS, M. A. Princípios da Medicina de Família: quatro pilares que definem sua identidade. O Mundo da Saúde, v. 34, n. 3, p. 300-310, 30 set. 2010.

MENDES, E. V. As redes de atenção à saúde. Ciência \& Saúde Coletiva, v. 15, n. 5, p. $2297-$ 2305, ago. 2010.

PIMENTEL, Í. R. S. et al. Caracterização da demanda em uma Unidade de Saúde da Família. Revista Brasileira de Medicina de Família e Comunidade, v. 6, n. 20, p. 175-181, 11 ago. 2011.

RUBEM DAVID AZULAY; DAVID RUBEM AZULAY; AZULAY-ABULAFIA, L. Dermatologia. Rio De Janeiro (Rj): Guanabara Koogan, 2008. 